\title{
Feasibility of Particle Imaging Velocimetry in Cone Calorimeter experiments
}

\author{
SIMON DELCOUR ${ }^{1,2}$, DAMIEN HEBERT ${ }^{3}$, FRANCOIS-XAVIER OUF ${ }^{1}$, ALEXIS COPPALLE $^{3}$, \\ NATHALIE AZEMA ${ }^{2}$, LAURENT FERRY ${ }^{2}$, JOSE-MARIE LOPEZ-CUESTA ${ }^{2}$, FRANCOIS SALM ${ }^{1}$, \\ MARTINE TALBAUT ${ }^{3}$ and JEROME YON ${ }^{3}$ \\ ${ }^{1}$ Institut de Radioprotection et de Sûreté Nucléaire (IRSN), Laboratoire de Physique et de Métrologie des \\ Aérosols, B.P. 68, 91192 Gif-Sur-Yvette Cedex, France. \\ ${ }^{2}$ Ecole des Mines d'Alès, Centre des Matériaux (C2MA)-Pôle Matériaux Polymères Avancés, 6 Avenue de \\ Clavières, 30319 Alès Cedex, France. \\ ${ }^{3}$ Complexe de Recherche Interprofessionnel en Aérothermochimie, CNRS UMR 6614, Université de \\ Rouen, site universitaire du Madrillet, BP 12, 76801 Sant Etienne du Rouvray Cedex, France.
}

\begin{abstract}
Experimental results are reported on the feasibility of particle imaging velocimetry in a cone calorimeter. PolyMethyl MethAcrylAte (PMMA) plates have been contaminated at their surface by alumina particles $\left(D_{\mathrm{ev}}=4.4 \mu \mathrm{m}\right)$ as seeding particles allowing PIV analysis. Particles are released by bubble bursting during the thermal degradation of the PMMA for each heat flux investigated $\left(25,35\right.$ and $\left.45 \mathrm{~kW} / \mathrm{m}^{2}\right)$. A "layer" of particles could be noticed for the highest heat flux $\left(35\right.$ and $\left.45 \mathrm{~kW} / \mathrm{m}^{2}\right)$ while for the smallest flux $(25$ $\mathrm{kW} / \mathrm{m}^{2}$ ), the release is confined and more erratic. Velocity increases as a function of the height above the polymer surface from $0.03 \mathrm{~m} / \mathrm{s}$ at the PMMA surface to $0.3 \mathrm{~m} / \mathrm{s}$ at the bottom of the conical heater and these results are in good agreement with previous experiments and numerical simulations. As an overall conclusion, this first attempt demonstrates the huge potential of PIV analysis, associated to our deposition set-up, for investigating flow field during cone calorimeter experiments involving bubbling polymers.
\end{abstract}

KEYWORDS: fluid dynamics, PIV, velocity, cone calorimeter, flow field, convective flow

\section{NOMENCLATURE LISTING}

$\begin{array}{ll}\mathrm{Al}_{2} \mathrm{O}_{3} & \text { aluminium dioxide or alumina } \\ D_{e v} & \text { mean equivalent volume diameter }(\mu \mathrm{m}) \\ \mathrm{Kn} & \text { Knudsen number } \\ P I V & \text { Particle Imaging Velocimetry } \\ P M M A & \text { PolyMethyl MethAcrylate } \\ \mathrm{Re}_{p} & \text { Reynolds number of the particle } \\ S / N & \text { signal to noise ratio } \\ t_{0} & \text { time reference } \\ y & \text { height above PMMA }(\mathrm{mm})\end{array}$

$\begin{array}{ll}\text { Greek } & \\ \lambda & \text { mean free path of the gas }(\mu \mathrm{m}) \\ \mu_{\text {air }} & \text { dynamic air viscosity }(\mathrm{Pa} . \mathrm{s}) \\ \rho_{\text {air }} & \text { air density }\left(\mathrm{kg} / \mathrm{m}^{3}\right) \\ \rho_{\text {part }} & \text { particle density }\left(\mathrm{kg} / \mathrm{m}^{3}\right) \\ \sigma_{\mathrm{g}} & \text { geometric standard deviation } \\ \tau_{\mathrm{p}} & \text { relaxation time of the particle (s) }\end{array}$

\section{INTRODUCTION}

The cone calorimeter (ASTM 1354, ISO 5660) is a widespread experimental apparatus for the determination of the fire behavior of various materials. It provides information on the time to ignition (either auto-ignition or using an igniter), Heat Release Rate (HRR), soot and gases ( $\mathrm{CO}$ and $\mathrm{CO}_{2}$ ) yields. It can be associated to additional experimental equipment like Fourier Transform Infra-Red spectrometer (FTIR) to obtain a better insight on the chemical products of the thermal degradation of materials.

Recent studies were conducted in order to validate some critical hypotheses originally made on the thermal behavior of this apparatus [1]. The black-body hypothesis for the radiant panel was confirmed [2] and a thorough investigation of the convective exchange on the sample surface was performed [3].

The flow patterns in the area between the radiant heater and the sample (identified hereafter as the degradation area) is often assumed to be the textbook case of natural convection on a horizontal plate. This flow was either measured with the radiant panel turned off or modeled through Computational Fluid Dynamics methods [4][5] but so far, no experimental data was available on the flow field inside this degradation area during the thermal degradation of a solid sample. 
Particle Imaging Velocimetry (PIV) is a well-known measurement technique in fluids mechanics, widely used to provide a complete two-dimensional (or even three-dimensional) flow image, allowing insight of the flow structure. In fire science, it has been commonly used to investigate into the flow field through vents (like doors and windows) in compartment fire tests [6] or in open vegetation fires [7]. However, PIV measurement in a standard, lab-scale apparatus like the Cone Calorimeter was never performed. Using PIV in a fire environment implies the overcoming of two major difficulties: the choice of the seeding particles and the choice of the seeding system.

We propose here an experimental method to provide a complete description of the flow field in the degradation area. Classical PIV approaches imply the injection of particles in the gaz flow. The originality of the proposed method is that seeding particles are previously deposited on the solid material and released during the degradation phenomenon, through bubbling. Using this method, we are able to generate a good seeding of the degradation area, especially in the area close to the fuel surface, where the air flow will interact with the pyrolizing material.

Previous studies in the field of nuclear safety [8][9][10][11], regarding the re-suspension of solid particle from burning substrates have identified a specific behavior of particles on Poly-Methyl Methacrylate (PMMA), namely a "peak" of particles emission at the beginning of degradation (prior to flaming). This peak of emission was used in the present work as the seeding mechanism for PIV measurements in a cone calorimeter. A specific deposition device, based on the inertial impaction mechanism, was designed and used to produce well defined particle deposits at the surface of PMMA plates.

The results obtained showed that PIV will allow a better comprehension of the flow field in the degradation area. The current setup is not potent enough to obtain a precise representation of the mixing layer where the pyrolysis gases mix with air, but we were able to give more insight on this area nonetheless. Among potential application, we could name nuclear safety applications, nanoparticlespolymers composites emission rate measurement [12] and fundamental study of pyrolysis interaction with air flow.

\section{PARTICLE DEPOSITION SYSTEM}

\section{Base concept and apparatus design}

The main objective of the device was to generate a dry, spatially homogeneous deposit of solid particles with user control of the particle size and deposition density. Classical solvent-coating methods were excluded because of the possible bias on the adhesive bonding between the particles and the substrate. Within these constraints, gravitational settling and inertial impaction were the only processes that could be applied. Inertial impaction was chosen because it is faster than gravitational settling.

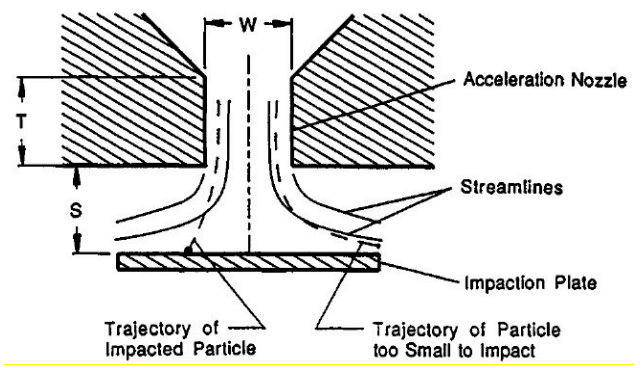

Fig. 1. Streamlines and particle trajectories for a typical impactor [13].

The base principle of inertial impaction is presented in Fig. 1 [13]. Historically, this principle was used in multi-stage impactor (known as cascade or Andersen impactor [14]) to obtain an inertial classification of the sampled aerosol. This kind of device is characterized through an "S-shaped" deposit efficiency curve, based on particle inertia. Particle large enough will drift from the streamlines and impact on the plate while particle small enough will follow the streamlines and exit the system. 


\section{Materials and methods}

The polymer used in this study was clear PMMA (PMMA 1.18, Delire SA, similar to the one used in [8]). Samples were square plates, $100 \mathrm{~mm}$ wide and $8 \mathrm{~mm}$ thick, table 1 presents the properties of this material. This polymer is known to form bubbles when undergoing thermal degradation. For this study, alumina particles (SPM 102, Alumines Durmax S.A.) were selected, because of their excellent thermal stability and their availability in different sizes and shapes. The powder was dried for at least two hours at $120^{\circ} \mathrm{C}$ before being used.

Table 1. Material properties.

\begin{tabular}{|l|c|}
\hline Density & $1190 \mathrm{~kg} / \mathrm{m}^{3}$ \\
\hline Specific heat & $1460 \mathrm{~J} / \mathrm{kg} / \mathrm{K}$ \\
\hline $\begin{array}{l}\text { Thermal } \\
\text { Conductivity }\end{array}$ & $0.188 \mathrm{~W} / \mathrm{m} / \mathrm{K}$ \\
\hline $\begin{array}{l}\text { Glass transition } \\
\text { temperature }\end{array}$ & $118^{\circ} \mathrm{C}$ \\
\hline $\begin{array}{l}\text { Auto ignition } \\
\text { temperature }\end{array}$ & $430^{\circ} \mathrm{C}$ \\
\hline
\end{tabular}

The basic impaction technique was developed into a device combining several throat impactor (to accommodate different particle sizes) and a moving impaction plate (PMMA plate) presented in Fig. 2. The entire deposition bench is also shown, including aerosol generator, filtration system and vacuum pump.
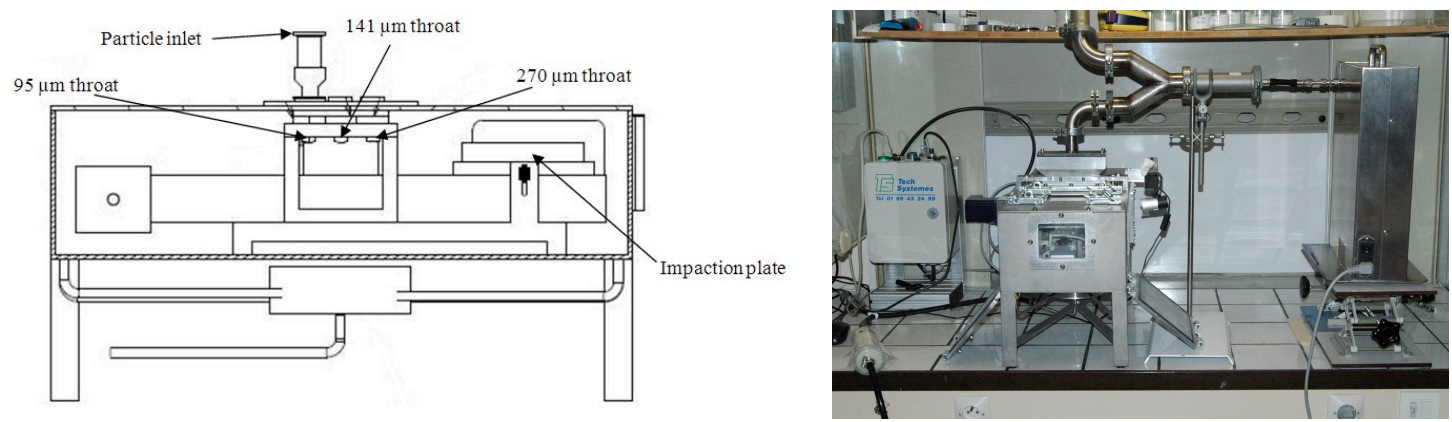

Fig. 2. Schematic side view of the impaction device (left) and front view of the deposition bench (right).

The appropriate slit is chosen, based on the volume equivalent median diameter of the particles, as well as the linear velocity of the impaction plate, based on the desired deposit surface density. In order to assess the amount of particles deposited on the surface, the PMMA plates are cleaned with ethanol and weighted prior to the introduction into the sample holder.

A rotary brush generator $\left(\mathrm{RBG}_{1000,} \mathrm{PALAS}()\right.$ ) is used to generate an aerosol of solid particles which is pumped through the slit by the vacuum pump. The volume flow through the selected throat is adjusted to $40 \mathrm{NL} / \mathrm{min}$. Once the flow of particles through the throat is stabilized $(\approx 20 \mathrm{~s})$, the movement of the impaction plate is initiated. At the end of the deposition operation, the sample is extracted and weighted again to determine the mass of the deposit.

\section{Characterization of the deposit}

The mean deposited mass was $87.7 \pm 7.7 \mathrm{mg}$ (standard deviation on 23 samples). Tests were also conducted with non-dried powder, showing that the presence of uncontrolled moisture in the powder tends to increase the mass of the deposit $(121 \pm 8.3 \mathrm{mg})$.

To evaluate the deposited particles' diameter, samples with deposits were washed with ISOTON electrolyte solution and the size distribution was measured with a Coulter apparatus (Multisizer 4, 
Beckmann Coulter( $)$. The size distribution is shown in Fig. 3, with raw powder size distribution for comparison. A fairly good agreement could be noticed between the size distributions of the raw aerosol and the deposited particles. The equivalent volume diameter $D_{\mathrm{ev}}$ is $4.4 \mu \mathrm{m}$ with a geometric standard deviation $\sigma_{\mathrm{g}}=1.3$ (assuming a log-normal size distribution). One must notice that these particles are similar to the one previously used by Ouf et al. [8] for investigating the release of particles deposited at the surface of a burning polymer.
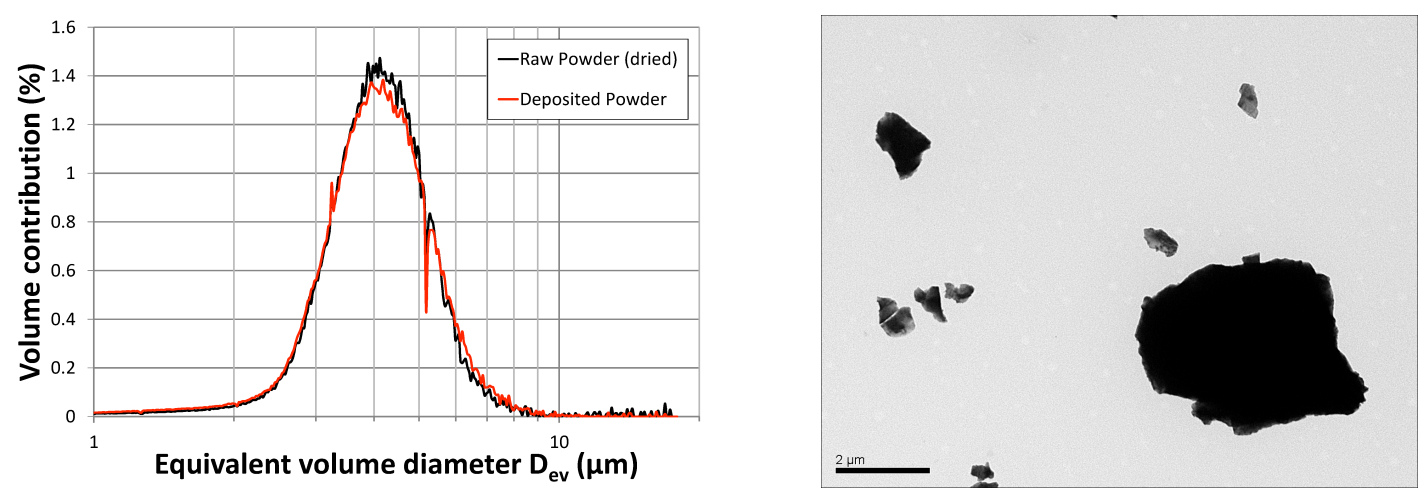

Fig. 3. Size distribution of raw powder and deposited particles (Coulter measurements) and TEM image of the raw powder.

Surface coverage was investigated with microscopic observations. Twelve pictures were taken in random areas of the samples and the surface coverage was measured with a threshold technique. Surface coverage was $85 \pm 2.1 \%$ except in two, $5 \mathrm{~mm}$ wide bands on the edges on the plates where it was $69 \pm 2.06 \%$. These bands were probably caused by flow instabilities at the edges of the samples.

Microscopic observation shows that the deposit is homogeneous, with very few agglomerates present on the surface. Given the mass of the deposit and size of the particles, it can be assumed that the deposit consists of a single layer of particles. This allows the assumption that the deposit will not hinder the thermal degradation by providing an insulating barrier against external heat flux.

\section{PIV SETUP}

The experimental setup for PIV experiments is shown in Fig. 4. This setup includes a double pulse NdYAG laser (532 nm, Evergreen Quantel) with lenses and mirrors to generate the laser sheet and direct it into the degradation area. Images acquisition is achieved with the help of two cameras (Imager Pro X 4M La Vision) with a resolution of $2048 \times 2048$ pixels, respectively associated with $50 \mathrm{~mm}$ and $135 \mathrm{~mm}$ objectives. For technical reasons, the radiant panel used in this study is not a standard cone calorimeter radiant heater but was extracted from a standard smoke chamber (ISO 5659). This radiant heater is roughly $30 \%$ smaller than the cone calorimeter version, but this should not modify greatly the resuspension phenomenon [8] which produces the seeding. This also implies that the mass loss of the sample could not be recorded.

The distance between the radiant heater and the sample is set to $25 \mathrm{~mm}$. The incoming heat flux on the sample is regulated by assigning the temperature of the heater and measuring the radiant flux with a heat flux gauge, prior to the degradation tests. This provides a calibration curve used latter on to set the desired heat flux on the sample. Three different heat fluxes were used, namely 25,35 and $45 \mathrm{~kW} / \mathrm{m} 2$, corresponding heater temperatures were respectively 560,655 and $720^{\circ} \mathrm{C}$.

Once the required temperature is reached, the sample is placed in a steel holder and put under the radiant heater, protected by a thermal screen (steel plate and ceramic). The start of the test, marked as t0, correspond to the removal of this protective screen. 


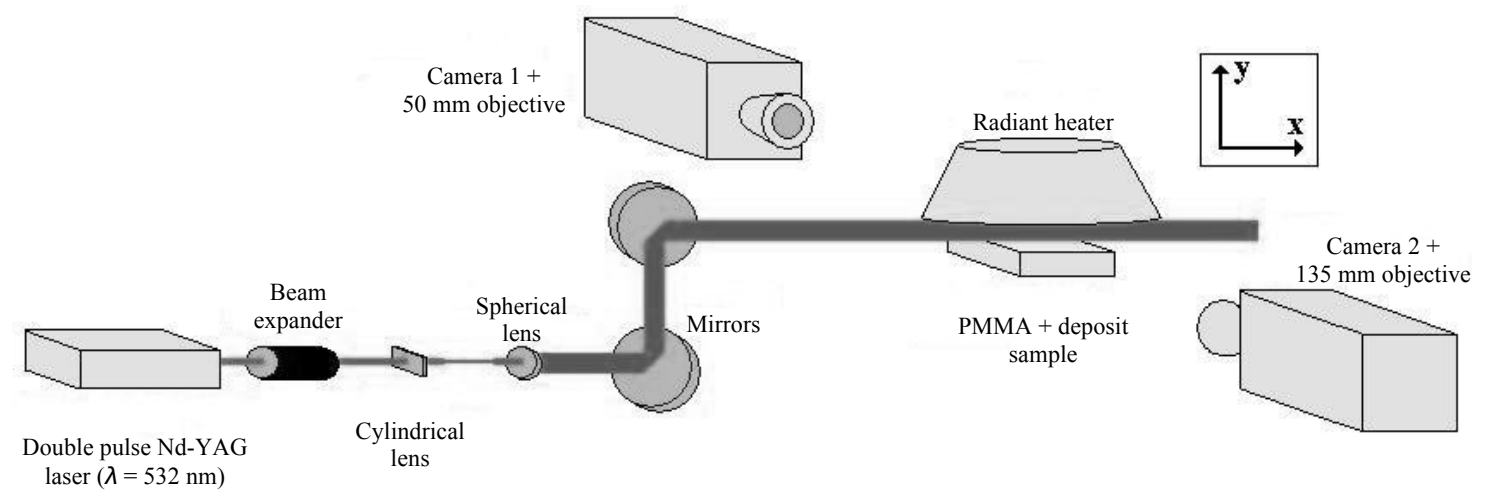

Fig. 4. Sketch of the experimental PIV setup.

Camera 1 is equipped with a $50 \mathrm{~mm}$ objective, providing a $113.9 \mathrm{~mm}$ wide field of view, with a resolution of $55.6 \mu \mathrm{m}$ per pixel. This field allows seeing the entire sample. Camera 2 is equipped with a $135 \mathrm{~mm}$ objective, providing a $46.5 \mathrm{~mm}$ wide field of view, with a resolution of $22.7 \mu \mathrm{m}$ per pixel. This field is centered on the axis of the radiant panel. Both fields were calibrated with a standard pattern. No interferential filter was used in this setup; therefore all observations will be realized before ignition, to avoid damages of the camera. When the sample ignites, the acquisition is stopped, the protective screen is put back in place and the flame is blown out with clean air.

The cameras were set on the double frame mode with $800 \mu$ s between the two frames. The global PIV system has a $5 \mathrm{~Hz}$ acquisition frequency. One commercial HD camera (HandyCam Sony HDR-SR11) was used for recording the overall experiments. Table 2 displays the recording conditions for the three tested heat fluxes, with $\mathrm{t}_{0}$ indicating the removing of the protection screen between the heater and the sample. The beginning of the recording has been considered as the first visual appearance of ejection of particles.

Table 2. Recording conditions.

\begin{tabular}{|c|c|c|c|}
\hline $\begin{array}{c}\text { Heat Flux } \\
\left(\mathbf{k W} / \mathbf{m}^{\mathbf{2}}\right)\end{array}$ & $\begin{array}{c}\text { Start of recording } \\
(\mathbf{s})\end{array}$ & Recording length (s) & $\begin{array}{c}\text { Number of } \\
\text { frames }\end{array}$ \\
\hline 25 & $\mathrm{t}_{0}+120$ & 120 & 600 \\
\hline 35 & $\mathrm{t}_{0}+70$ & 60 & 300 \\
\hline 45 & $\mathrm{t}_{0}+40$ & 40 & 200 \\
\hline
\end{tabular}

\section{EXPERIMENTAL RESULTS: RAW IMAGES}

For convenience, an example of raw image is presented in Fig. 5 and positions of the conical heater, polymer surface, alumina deposit and laser sheet are mentioned. Due to their size and optical properties, the alumina particles deposit is easily visible at the polymer surface during PIV and particles ejection could be tracked and investigated.

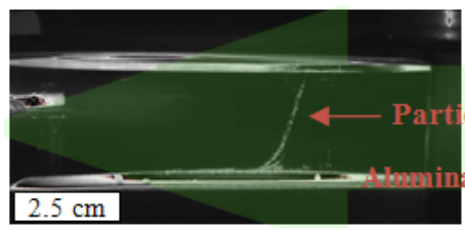

$\mathrm{t}_{0}+45 \mathrm{~s}$

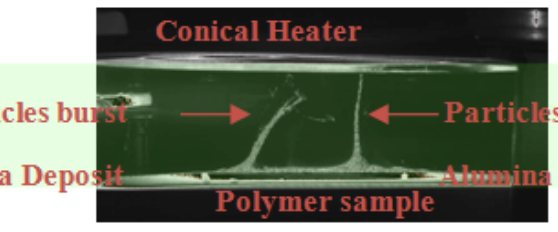

$\mathrm{t}_{0}+55 \mathrm{~s}$

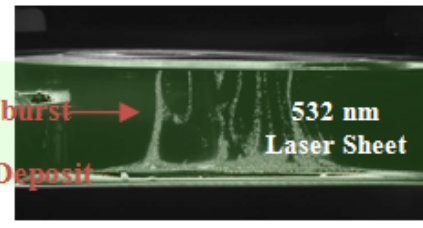

$\mathrm{t}_{0}+65 \mathrm{~s}$

Fig. 5. Raw PIV images as a function of time for a contaminated polymer sample under $45 \mathrm{~kW} / \mathrm{m}^{2}$ heat flux (camera 1 with $50 \mathrm{~mm}$ objective).

Raw PIV images, obtained with the $135 \mathrm{~mm}$ objective, are presented in table 2 for different times since the application of the heat flux. Prior to the "peak of emission", identified on the HD camera, no particles are ejected from the polymer. For the contaminated polymers and after the time duration corresponding to 
the "peak of emission", particles are ejected and this release is not homogeneous over the polymer surface. Similar experiments have been carried out for non-contaminated polymer samples and these particle "plumes" have not been reported. Then, the "peak of emission" identified on HD camera and PIV images could be associated to the alumina particles deposited at the polymer surface. Consequently, the end of this "peak of emission" is associated to the absence of particles at the surface of the melted polymer, demonstrating that such resuspension mechanism is extremely efficient.

For the lowest heat flux $\left(25 \mathrm{~kW} / \mathrm{m}^{2}\right)$, the release is confined and erratic, and filamentary structures are observed. Pictures show that the direction of the filaments may be sometimes oblique. Thus all the ejections of particles are not illuminated by the laser sheet in each instantaneous image. The ejection of particles is not continuous but occurs only from some spots on the PMMA surface. On each instantaneous picture, it has been observed that the size of ejection zone is about a few mm above the PMMA surface and the size of the ejected filament (in the horizontal direction) becomes thinner (about $1 \mathrm{~mm}$ ). For higher heat fluxes $\left(35\right.$ and $45 \mathrm{~kW} / \mathrm{m}^{2}$ ), the size of the ejection zone increases, producing larger ascendant flows of particles. One could identify, for $35 \mathrm{~kW} / \mathrm{m}^{2}$ and $45 \mathrm{~kW} / \mathrm{m}^{2}$, respectively at $\mathrm{t}_{0}+100 \mathrm{~s}$ and $\mathrm{t}_{0}+66 \mathrm{~s}$, a layer of re-suspended particles approximately $1 \mathrm{~mm}$ high.

Table 2. Evolution of raw PIV images (camera 2) as a function of heat flux and time since its application.

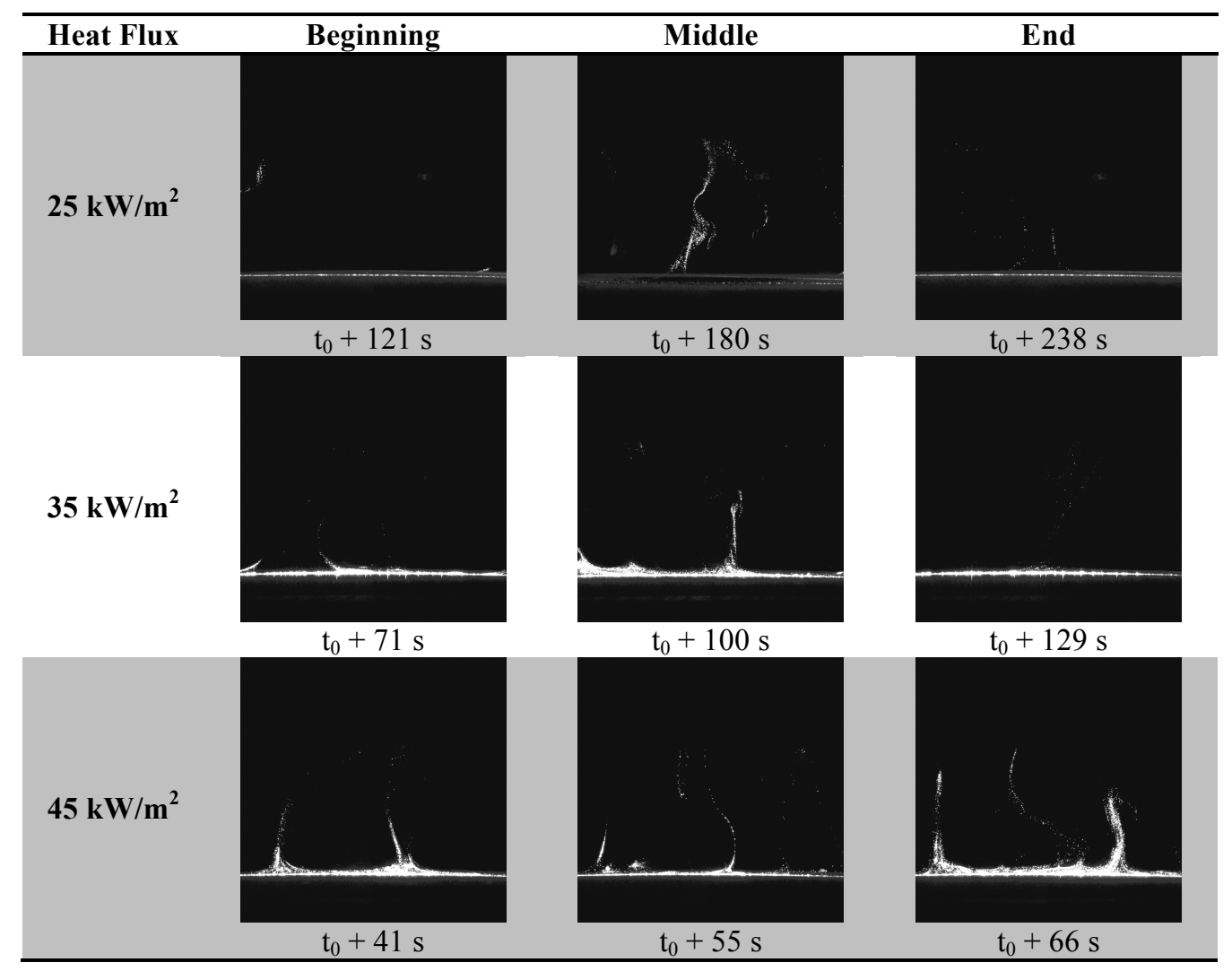

In this case, one could assume that particles are re-suspended from the surface by bubbles inside the viscous films at the top of the PMMA slab, due to pyrolysis of the polymer [8][9]. Then, the re-suspended particles are entrained from this layer by the convective flow through turbulent bursts, similar to the ones described by Cleaver and Yates [15] and more recently by Zhu et al. [16]. An illustration of the physical phenomenon, involved during release of alumina particles, is presented in figure 6 . Due to the high luminosity of the layer containing the bubbles and the re-suspended particles (see figure 5 and table 3), and due to the short time during which the bubbles remain at the PMMA surface (10-30 ms), we were not able to observe the formation of such bubbles with the help of the present PIV set-up. Further experiments 
will be performed with a micro-PIV set-up at higher frequency (100 Hz to $1 \mathrm{kHz})$ to record ejection of particles from a single bubble.

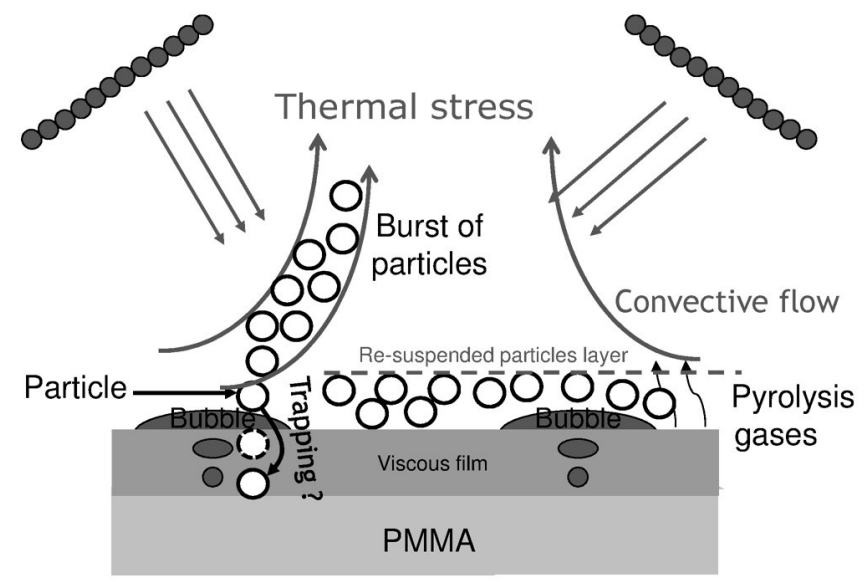

Fig. 6. Schematic illustration of particle release during thermal degradation of PMMA.

\section{EXPERIMENTAL RESULTS: PIV ANALYSIS}

Velocity vectors have been computed with an adaptive PIV method (DynamicStudio, Dantec ${ }^{\circledR}$ ). Three sizes of windows (64x64 pixels, $48 \times 48$ pixels and $32 \times 32$ pixels) have been considered for determining correlation peak. Two filters have been applied to the corresponding peak of correlation, the first one eliminating peak with low $\mathrm{S} / \mathrm{N}$ ratio (lower than 6 ), the second one by rejecting velocity vector higher than a threshold of $0.4 \mathrm{~m} / \mathrm{s}$.

With the PIV measurements, the factors entering into the errors are particle image size, size of the interrogation window, local velocity gradients, number of particles within the sampling window and instrumentation performance characteristics (camera and digitizer). As shown by Willert and Gharib [17] sub-pixel displacements can be determined. An overall estimation of the velocity uncertainty is given by the repeatability of the experimental set-up. In this study, it was not possible to repeat many times the degradation of a PMMA sample. If we consider, as Franck et al. [18], that the standard deviation of the PIV measurements under the same experimental conditions, is about 0.1 pixel, this corresponds to an uncertainty equal to $0.007 \mathrm{~m} / \mathrm{s}$ for the wide field of view (camera 1) and $0.003 \mathrm{~m} / \mathrm{s}$ for the small one (camera 2).

The spatial response of the PIV is influenced by the size of the interrogation window. This effect results in a spatial filter with a high frequency cut-off [19]. With the ultimate size $32 * 32$ pixels of the interrogation window, spatial variations of the velocity less than $0.7 \mathrm{~mm}$ cannot be measured.

Table 4 presents the flow field associated to the raw images of table 3. It is obvious that the velocities have been determined only inside the particles-loaded filamentous structures. Outside these filaments, no particle signals have been detected by the cameras. The main feature of these results is that the particle speed is low close to the PMMA surface, less than $0.03 \mathrm{~m} / \mathrm{s}$, and it increases with height to reach a value close to $0.3 \mathrm{~m} / \mathrm{s}$. The flow fields recorded at each frame of the PIV recording $(600,300$ and 200 frames respectively for 25,35 and $45 \mathrm{~kW} / \mathrm{m}^{2}$ ) have been summed and the resulting mean flow fields are presented in Fig. 7. One must notice that in this case, the overall field between the PMMA surface and the bottom of the conical heater has been covered by the seeding particles, allowing us the reconstruction of a complete flow field. According to these mean results, several conclusions could be drawn:

- taking into account the velocity uncertainty described by Franck et al. [18], the velocity in the resuspended particles layer $(0<\mathrm{y}<5 \mathrm{~mm})$ is not sensitive to the heat flux,

- the height of this layer increases with the heat flux,

- the velocity close to the conical heater $(17<\mathrm{y}<25 \mathrm{~mm})$ increases with the heat flux. 
Table 4. Evolution of the flow field as a function of heat flux and time since its application.

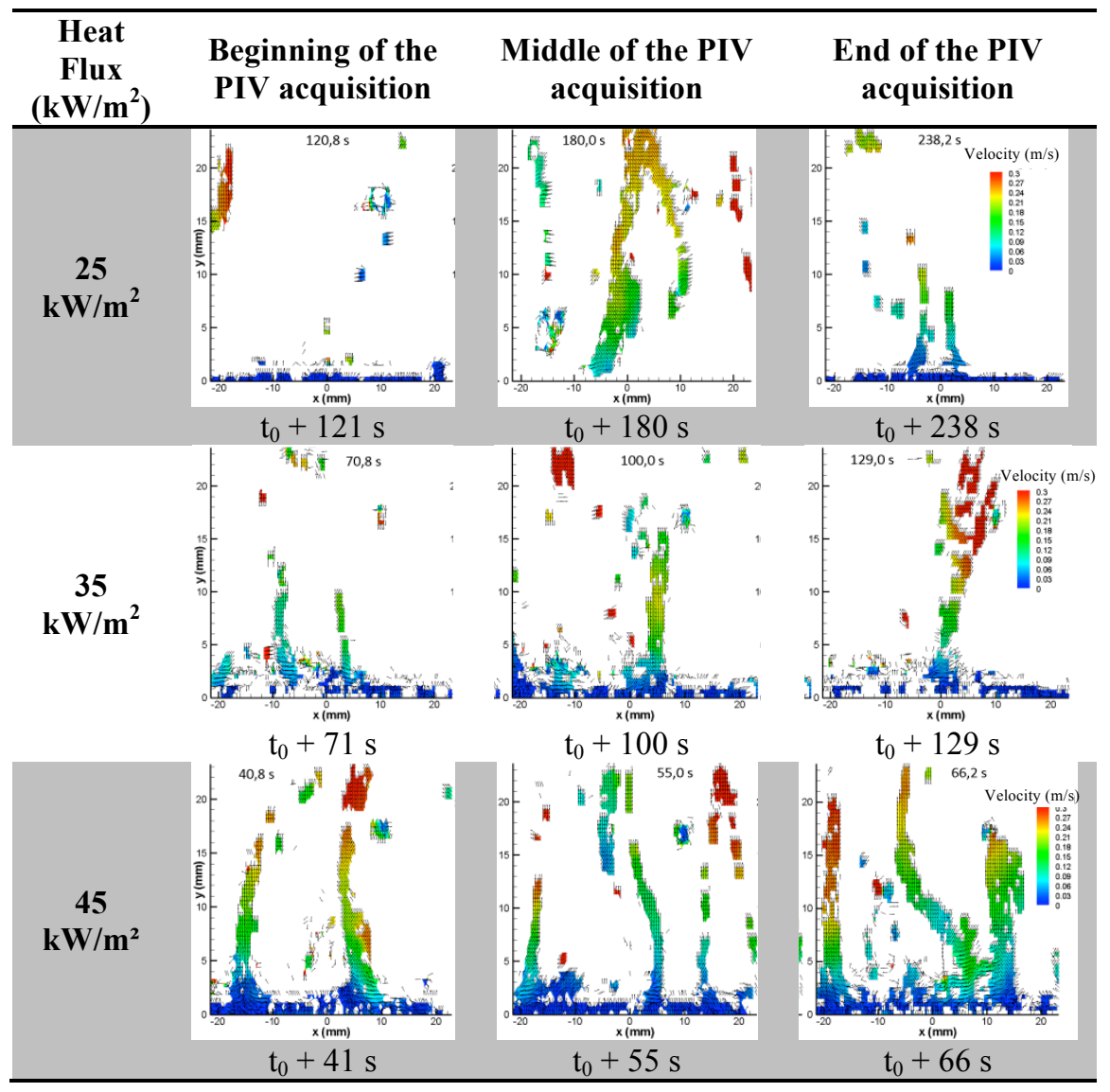

The Knudsen number $\mathrm{Kn}=2 \lambda / \mathrm{D}_{\mathrm{ev}}$, with $\lambda$ being the mean free path of air molecules $(0.0674 \mu \mathrm{m}$ for air at normal temperature and pressure), it is smaller than $10^{-2}$ with the mean particle size $\mathrm{D}_{\mathrm{ev}}=4.4 \mu \mathrm{m}$. So, the particle exchanges with air are in the continuum regime. The particle Reynolds number $\operatorname{Re}_{\mathrm{p}}$ is defined as $\rho_{\text {air }} \mathrm{V} \mathrm{D}_{\text {ev }} / \mu_{\text {air }}$, with $\mathrm{V}$ being the characteristic speed of the particle and $\mu_{\text {air }}$ the viscosity of air. With $\mathrm{V}$ equal to $0.3 \mathrm{~m} / \mathrm{s}, \mathrm{Re}_{\mathrm{p}}$ is smaller than unity and the Stokes' drag law is considered to apply. Consequently, the characteristic particle response time $\tau_{\mathrm{p}}=\rho_{\mathrm{part}} \mathrm{D}_{\mathrm{ev}}{ }^{2} /\left(18 \mu_{\text {air }}\right)$ is about $0.2 \mathrm{~ms}$ (with $\rho_{\text {part }}=410^{3} \mathrm{~kg} / \mathrm{m}^{3}$ and $\left.\mu_{\text {air }}=2610^{-6} \mathrm{~Pa} . \mathrm{s}\right)$. The characteristic convective time is given by the distance between the PMMA sample and the bottom of the cone, and is equal to $83 \mathrm{~ms}$, i.e. much bigger than $\tau_{\mathrm{p}}$, which indicates that the particles are well entrained by the convective flow. 

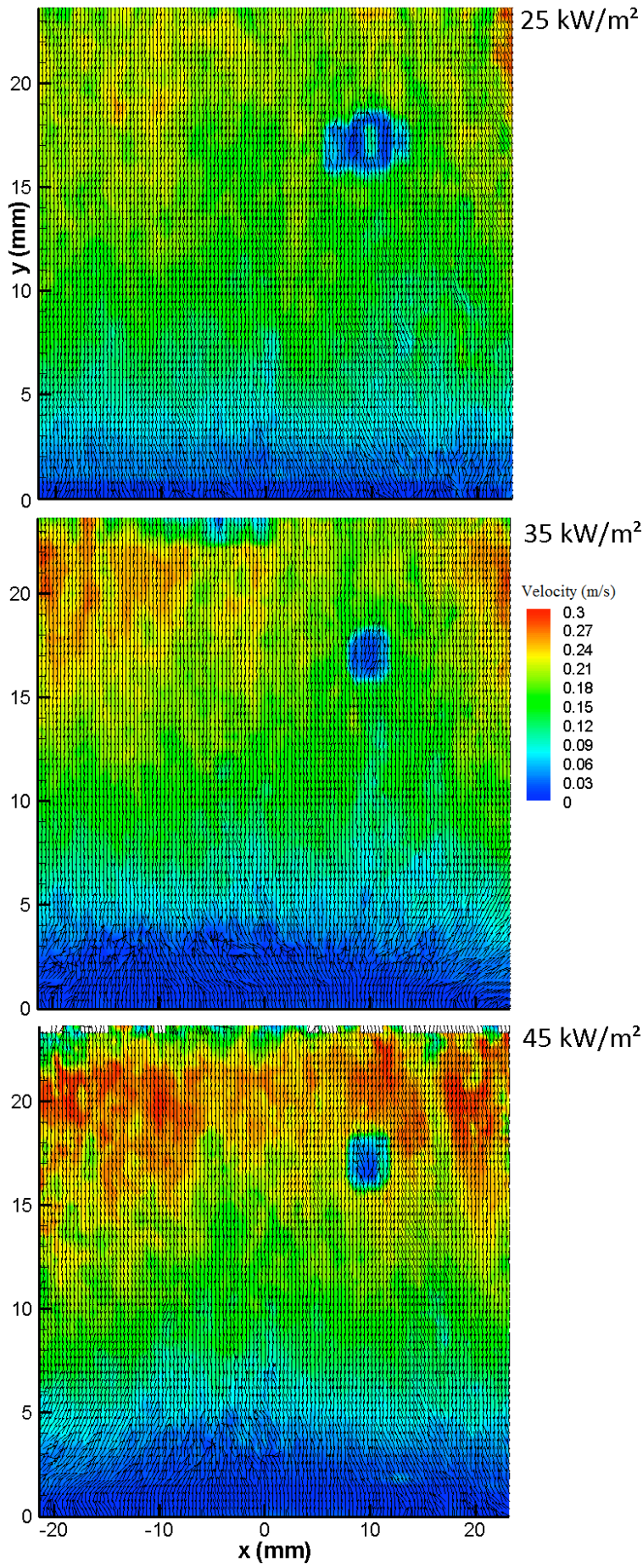

Fig. 7. Mean flow fields as a function of heat flux (camera 2 with $135 \mathrm{~mm}$ objective).

Figure 8 presents the mean flow field for the entire conical heater (camera 1). The same conclusions could be drawn from these results and the confined convective flow, induced by the conical heater, is clearly identified for $45 \mathrm{~kW} / \mathrm{m}^{2}$. Between 25 and $35 \mathrm{~kW} / \mathrm{m}^{2}$ the characteristics of the re-suspended particles layer seem to increase (height and mean velocity). Beyond a heat flux of $35 \mathrm{~kW} / \mathrm{m}^{2}$, these characteristics seem to be constants. This point is of main interest for improving our comprehension of re-suspension kinetics of particles deposited at the surface [8][9][10][11] or integrated in the polymer matrix [12]. 


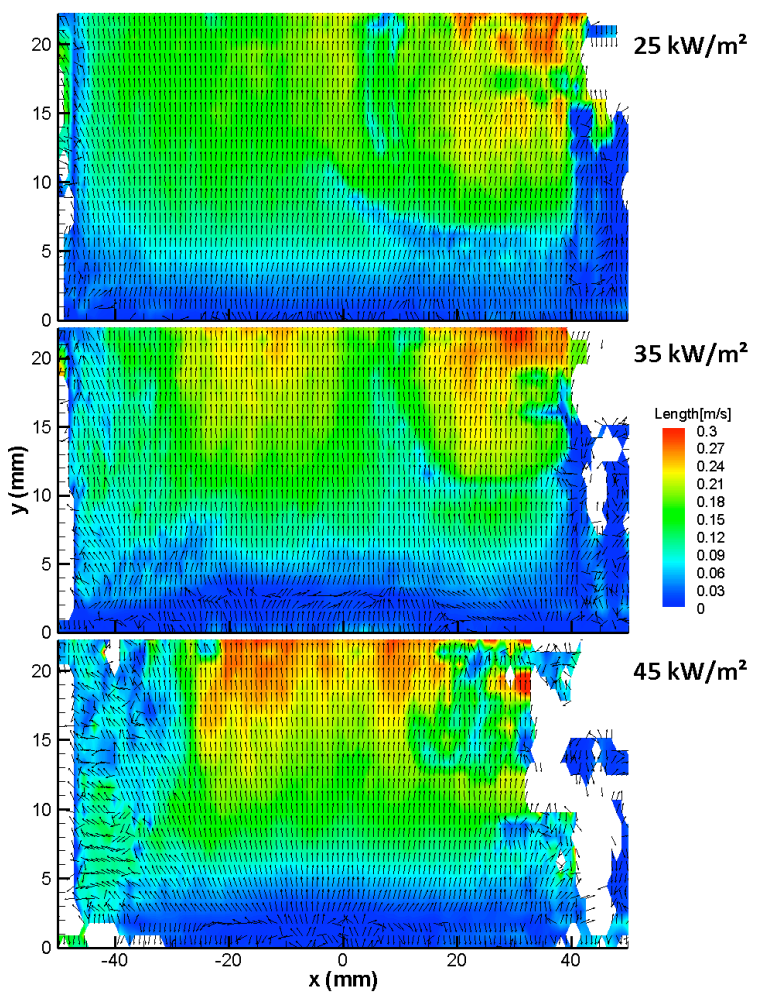

Fig. 8. Mean flow fields as a function of heat flux (camera 1 with $50 \mathrm{~mm}$ objective).

Figure 9 presents the significant evolution of the average velocity on the sample axis as a function of the height above the polymer surface $y$. For 35 and $45 \mathrm{~kW} / \mathrm{m}^{2}$ and close to the polymer surface $(\mathrm{y}<2.5 \mathrm{~mm})$, the average velocity does not denote a specific trend of evolution. Considering the experimental uncertainties $(0.003 \mathrm{~m} / \mathrm{s})$ it is not obvious that the velocity is fully constant in the region and further investigations are needed to confirm this first analysis.

At $y=22.5 \mathrm{~mm}$, corresponding to the conical heater, the average velocity is between 0.2 to $0.3 \mathrm{~m} / \mathrm{s}$, in agreement with numerical computation of Tsai et al. [5] who have reported average velocity ranging from 0.15 to $0.3 \mathrm{~m} / \mathrm{s}$. As a consequence, our results are in good agreement with previous attempts to compute the flow velocity in a cone calorimeter. One must also notice that experimental measurements by Tsai et al. [5] have been obtained without the conical heater because hot wire anemometry is not suitable within fire environment. In this case, PIV analysis, associated to our experimental procedure is a powerful technique, suitable to extreme conditions encountered in fire and combustion studies. 


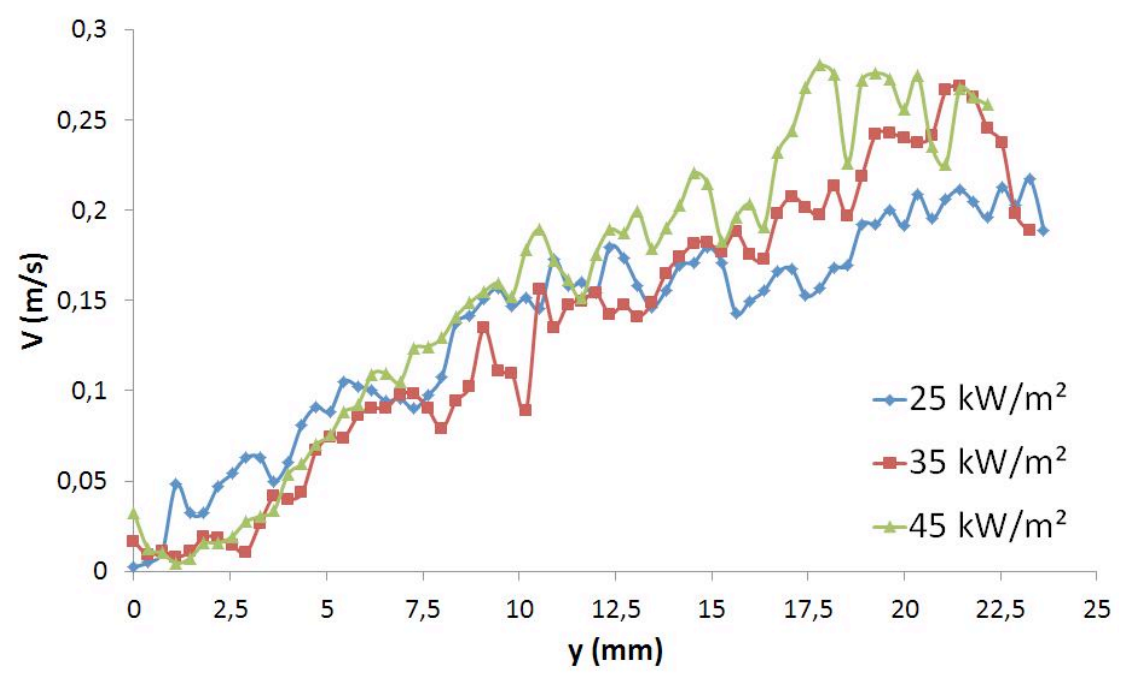

Fig. 9. Evolution of the average velocity as a function of height above the polymer surface.

One could discuss the validity of using an average in this case, as it is likely that the sample is not under steady state degradation conditions. If we consider our acquisition timeframe and the surface temperature data from [5], it appears that all our measurements were performed in regions where the surface temperature is not varying strongly (between 300 and $350^{\circ} \mathrm{C}$ ). As surface temperature is the critical parameter defining the convective flow far from the sample, this flow field will probably not exhibit a strong variation. This also explains why the velocities measured far from the sample are not dependent on the heat flux (except in the area near the heater, where the heater temperature and geometry becomes the governing parameter).

As the mass loss was not recorded, we used the literature data to evaluate the velocity close to the sample [20]. Assuming a mass loss rate of $10 \mathrm{~g} / \mathrm{m}^{2} / \mathrm{s}$ and a MMA vapor density of $4 \mathrm{~kg} / \mathrm{m}^{3}$ will yield an estimated value of velocity of $0.0025 \mathrm{~m} / \mathrm{s}$ at the surface, lower than our value of $0.03 \mathrm{~m} / \mathrm{s}$. This difference can be explained by the fact that our setup is sensitive enough to show the influence of bubble bursting. The theoretical computation assumes that the vapor emission is a homogeneous diffusion process, whereas the bubble bursting is very local phenomenon in space and time, inducing very high variations of air velocity. This phenomenon cannot be captured by a global measurement like mass loss rate.

Even if the current experimental setup is not precise enough to detect individual bubble bursting, it proved sensitive enough to highlight the fact that gas emission from PMMA undergoing thermal degradation is having an influence on the local air flow above the sample. Further work will be conducted to develop a high frequency micro-PIV setup, in order to investigate the area close to the polymer sample.

\section{CONCLUSIONS}

This study demonstrates the feasibility of the determination, by optical diagnostic, of the high resolved convective flow induced by cone calorimeters that are widely used for the study of materials thermal degradation. For this purpose a commercial PIV system has been used. The main experimental originality is the method used to inject the seeding particles. Indeed, these particles are homogeneously deposited on the surface of PMMA by a protocol described in this paper. Alumina particles are used but the protocol could be implemented for different size and nature of seeding particles. These particles are pulled up thanks to the bursting of bubbles induced by the PMMA degradation. This interesting phenomenon is also detailed. The convective flows were determined for three heat fluxes and are in good agreement with previous experiments and numerical simulations. The velocity appears to increase from $0.03 \mathrm{~m} / \mathrm{s}$ at the polymer surface to $0.3 \mathrm{~m} / \mathrm{s}$ at the bottom of the cone heater. Furthermore, for the highest heat flux, a "layer" of re-suspended particles has been identified and particles appear to be trapped in this layer before being extracted by convective flow through turbulent bursts. 
The results of this study open a wide new field of experimental investigations in fire science. The applicability of the technique allows the study of the turbulent mixing of air and pyrolysis gases, a critical step in the autoignition. Furthermore, the use of a more potent PIV setup (higher frequency and better spatial resolution) would allow the investigation of near surface interactions between the fuel and the gas phase. These data will also provide a reliable benchmark for CFD code validation, because of the nonintrusive nature of the measurement used.

Another potent evolution of this technique is to include the particles inside the polymer matrix (compounding). This way, the particle release could be in direct relation to the sample mass loss rate. Furthermore, this technique could be used to study the release of nanoparticles included in the burning material, potentially offering an evaluation of the source term in exposure assessment studies.

The instantaneous velocity fields also give a clear description of the bubbling dynamics. In further investigations, for understanding the release phenomenon, micro-PIV analysis at high frequency $(100 \mathrm{~Hz}$ to $1 \mathrm{kHz}$ ) will be performed on bubbles during their bursting. In this case, we will be able to record the acceleration of particles after the break-up of the bubbles and the elementary release efficiency of a single bubble. Further prospects will be to perform PIV analysis after the ignition. In addition to the deposition on the surface, the particles will also be implanted inside the polymer.

\section{REFERENCES}

[1] Schartel B., Bartholmai M., Knoll U., (2005) Some comments on the use of cone calorimeter data, Polymer Degradation and Stability 88: 540-547.

[2] Boulet P., Parent G., Acem Z., Rogaume T., Fateh T., Zaida J., Richard F., (2012). Characterization of the radiative exchanges when using a cone calorimeter for the study of the plywood pyrolysis, Fire Safety Journal 51: 53-60.

[3] Staggs J. E. J., (2011) A reappraisal of convection heat transfer in the cone calorimeter, Fire Safety Journal 46(3): 125-131.

[4] Brescianini C. P., Yeoh G.H., Chandrasekaran V., Yuen R., (1997) A Numerical Model for Pilot Ignition of PMMA in a Cone Calorimeter, Combustion Science and Technology 129(1-6): 321-345.

[5] Tsai, T.-H., M.-J. Li M.-J., Shih I.Y., Jih R., Wong S.-C., (2001) Experimental and numerical study of autoignition and pilot ignition of PMMA plates in a cone calorimeter, Combustion and Flame 124(3): 466-480.

[6] Bryant, R.A. "Particle image velocimetry measurements of buoyancy induced flow through a doorway". National Institute of Standards and Technology Report NISTIR 7252, Gaithersburg, MD, 2005, 71 p.

[7] Morandini F., Silvani X., Susset A. (2012) Feasibility of particle image velocimetry in vegetative fire spread experiments, Experiments in Fluids 53(1): 237-244.

[8] Ouf F.-X., Delcour S., Azema N., Coppalle A., Ferry L., Gensdarmes F., Lopez-Cuesta J.M., Niang A., Pontreau S., Yon J., (2013) Contribution to the study of particle resuspension kinetics during thermal degradation of polymers, Journal of Hazardous Materials 240-241: 298-307.

[9] Pickering S., (1986) A mechanism for the resuspension of particles from burning Plexiglas, Journal of Aerosol Science 17: 602-607.

[10] Buijs K., Chavane de Dalmassy B., Pickering S., (1989) The dispersion of radioactive aerosols in fire, Journal of Nuclear Materials 166: 199-207.

[11] Fernandez Y., Burghoffer P., (1995) Radioactive aerosols emissions in fires, Aerosol Science and Technology 23(2): 231-238.

[12] Nyden M.R., Harris R.H., Kim Y.S., Davis R.D., Marsh N.D., Zammarano M., "Characterizing particle emissions from burning polymer nanocomposites", 
Nanotechnology 2010: Advanced Materials, Cnts, Particles, Films And Composites Technical Proceedings Of The 2010 NSTI Nanotechnology Conference And Expo, NSTINanotech. 1, 2010, pp. 717-719.

[13] Baron P.A., Willeke K., Aerosol measurement - Principles, techniques and applications $\left(2^{\text {nd }}\right.$ ed), John Wiley \& Sons, New York, 2001, p. 237.

[14] Andersen A.A., (1958) New sampler for the collection, sizing, and enumeration of viable airborne particles, Journal of Bacteriology 76: 471-484.

[15] Cleaver J. W., Yates B., (1973) Mechanism of detachment of colloidal particles from a flat substrate in a turbulent flow, Journal of Colloid and Interface Science 44(3): 464-474.

[16] Zhu Y., Zhao B., Zhou B., Zhongchao T., (2011) A Particle Resuspension Model in Ventilation Ducts, Aerosol Science and Technology 46(2): 222-235.

[17] Wilbert C.E., Gharib M. (1991) Digital particle image velocimetry, Experiments in Fluids 10: 181-193.

[18] Frank J.H., Kalt P.A., Bilger R.W, (1999) Measurements of Conditional Velocities in Turbulent Premixed Flames by Simultaneous OH PLIF and PIV, Combustion and Flame 116:220-232.

[19] Foucaut J.M., Varlier J., Stanislas M. (2004) PIV optimization for the study of turbulent flow using spectral analysis, Measurement Science and Technology 15: 1046-1058.

[20] Rhodes B.T., Quintiere J.G., (1996) Burning Rate and Flame Heat Flux for PMMA in a Cone Calorimeter, Fire Safety Journal 26: 221-240. 